\title{
20 Inverted Precourses: Mathematikvorkurse an der Technischen Hochschule Ostwestfalen-Lippe
}

\section{Janina Stemmer}

Die Technische Hochschule Ostwestfalen-Lippe (TH OWL) bietet zum Start jedes Wintersemesters für zukünftige Studierende, die Wissenslücken in Englisch und naturwissenschaftlichen Fächern schließen möchten, betreute Präsenz- und Onlinekurse an. Diese Vorkurse bereiten gezielt auf die Anforderungen der Studiengänge vor. Sie helfen dabei, den eigenen Kenntnisstand zu ermitteln und bestehende Defizite auszugleichen. Diese freiwilligen Kurse finden unmittelbar vor Semesterbeginn an den drei Standorten der TH OWL sowie online auf der Lernplattform eCampus (ILIAS) statt und sind für immatrikulierte Studierende kostenfrei.

Im Jahr 2019 wurde ein neues Konzept für die Mathematikvorkurse eingesetzt, welches das individuelle und kollaborative Lernen stärken und vertieftes Wissen bei den Studienanfänger*innen fördern soll. Um die Teilnehmenden bei dem Erreichen ihrer Lernziele zu unterstützen, werden unter anderem verschiedene Aspekte des selbstregulierenden Lernens aufgegriffen. Die neuen Studierenden sollen von einer eher passiven, konsumierenden Haltung zu einer aktiven Rolle befördert werden, in welcher sie zunehmend Verantwortung für den eigenen Lernerfolg übernehmen. Der Ausbau dieser Fähigkeit ist für den positiven Verlauf der akademischen Ausbildung von großer Bedeutung.

Dafür wird die Blended-Learning-Methode Inverted Classroom eingesetzt. Als Inverted Classroom (oder auch Flipped Classroom), also „,vertauschter“ oder „,umgedrehter Klassenraum", wird ein didaktisches Konzept bezeichnet, in dem die grundlegende Wissensaneignung nicht während der Präsenzzeit stattfindet, sondern als Selbststudium vor die Präsenzveranstaltung gelegt wird. Durch die vorgelagerte Vermittlung von Grundlagen und die damit einhergehende Angleichung von Vorwissen bleibt in der Veranstaltung mehr Zeit, um gemeinsam Aufgaben zu erarbeiten, Fragen zu klären und Themen zu vertiefen. Durch das eigenständige Lernen in Kombination mit der ,vertiefende[n] Diskussion in der Präsenzphase werden Aspekte des aktivierenden Lernens, wie zum Beispiel Teamwork, Debatten und Selbstreflexion gefördert" (Tolks et al. 2016, S.14).

Für das Selbststudium vor der Präsenzveranstaltung erhalten die Studierenden Lernmaterialien mit dazugehörige Begleitfragen, welche sie zeit- und ortsunab-

Die Originalversion dieses Kapitels wurde revidiert. Ein Erratum ist verfügbar unter https://doi.org/10.1007/978-3-658-31279-4_32 
hängig sowie im eigenen Tempo und mit eigener Lernstrategie bearbeiten. Außerdem haben sie die Möglichkeit, online Unterstützung zu Lernstrategien oder zu fachlichen Inhalten in Lerngruppen und von E-Mentor*innen zu erhalten. Anschließend können die Teilnehmenden zu mehreren Zeitpunkten an betreuten Übungskursen teilnehmen. Hier werden Fragen beantwortet und das neu angeeignete Wissen durch (gemeinsame) Übungen gefestigt und vertieft. Um die vorherige Auseinandersetzung mit dem Lernstoff zu fördern, sollen die Teilnehmenden zuvor, angelehnt an das Just-in-Time-Teaching-Lehrkonzept, Fragen zu dem Lernstoff beantworten. Die Antworten dienen der Vorbereitung sowohl für die Studierenden als auch für die Lehrenden auf die Präsenzveranstaltung.

\subsection{Der umgedrehte Vorkurs}

Die Studierenden erhalten bereits acht Wochen vor Studienbeginn Zugang zu den Online-Materialien des Mathematikvorkurses. Diesem Material können sie sich in individueller Intensität und Geschwindigkeit widmen. Der Inhalt dient der Auffrischung von grundlegenden mathematischen Kenntnissen. Die Materialien bestehen aus sieben Lernzielorientierten Kursen (LoK) zu grundlegenden mathematischen Themengebieten:

- Arithmetik

- Funktionen

- Geometrie

- Gleichungen und Ungleichungen

- Mathematische Grundlagen

- Potenzen, Wurzeln, Logarithmen

- Trigonometrie

Aufgrund des sehr großen Umfangs der Materialien durchlaufen die Teilnehmenden zunächst den Diagnostischen Einstiegstest. Auf Grundlage der Testergebnisse erhalten die Studierenden Vorschläge zur Auswahl der Lernzielorientierten Kurse. Anschließend erfolgt ein weiterer Test in den Kursen, da die komplette Bearbeitung eines Kurses circa zwölf Stunden dauert. Somit erfahren die Studierenden zunächst, in welchen mathematischen Themengebieten sie ausreichende Kenntnisse aufweisen und wo Nachholbedarf besteht, und daraufhin, welche konkreten Themen sie im Vorkurs erarbeiten sollten. Die Lernzielorientierten Kurse setzen sich zusammen aus einem Eingangstest, drei bis fünf Lernzielen und einem Abschlusstest. Mit diesem können die Teilnehmenden überprüfen, wie erfolgreich ihr Selbststudium war. Die Lernziele beinhalten ausführliche Lernmodule sowie Trainingstests, mit Hilfe derer die zuvor rezipierten theoretischen Inhalte praktisch angewendet werden können. Der Vorkurs endet ebenfalls mit einem Abschlusstest, welcher Aussagen über den Lernfortschritt liefert. 


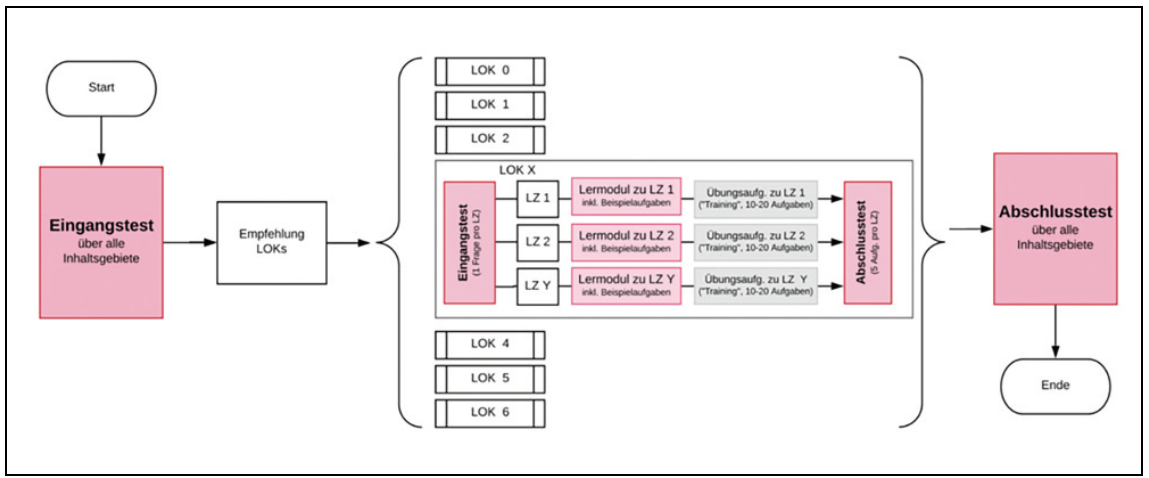

Abbildung 1: Flussdiagram zum Ablauf des Vorkurses

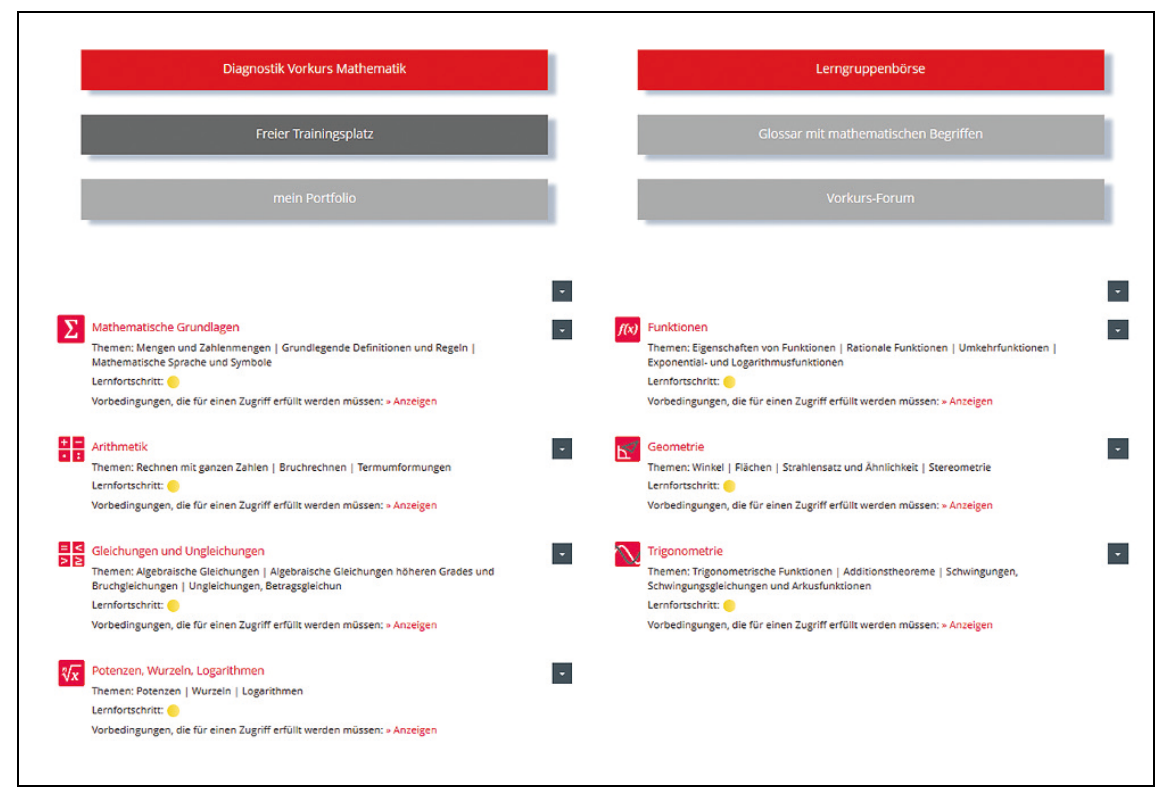

Abbildung 2: Übersichtsseite des Vorkurses auf der Lernplattform eCampus

Um sich die fachlichen Inhalte in diesem Blended-Learning-Szenarium anzueignen, müssen die Studierenden in der Lage sein, ihr Lernen selbst zu regulieren. Bedauerlicherweise weisen zahlreiche Lernende in dieser Fähigkeit Defizite auf, weshalb diverse Angebote zum Ausbau dieser Kompetenz konzipiert wurden (Bellhäuser und Schmitz 2013, S. 345). Aufgrund der großen Komplexität der Lernum- 


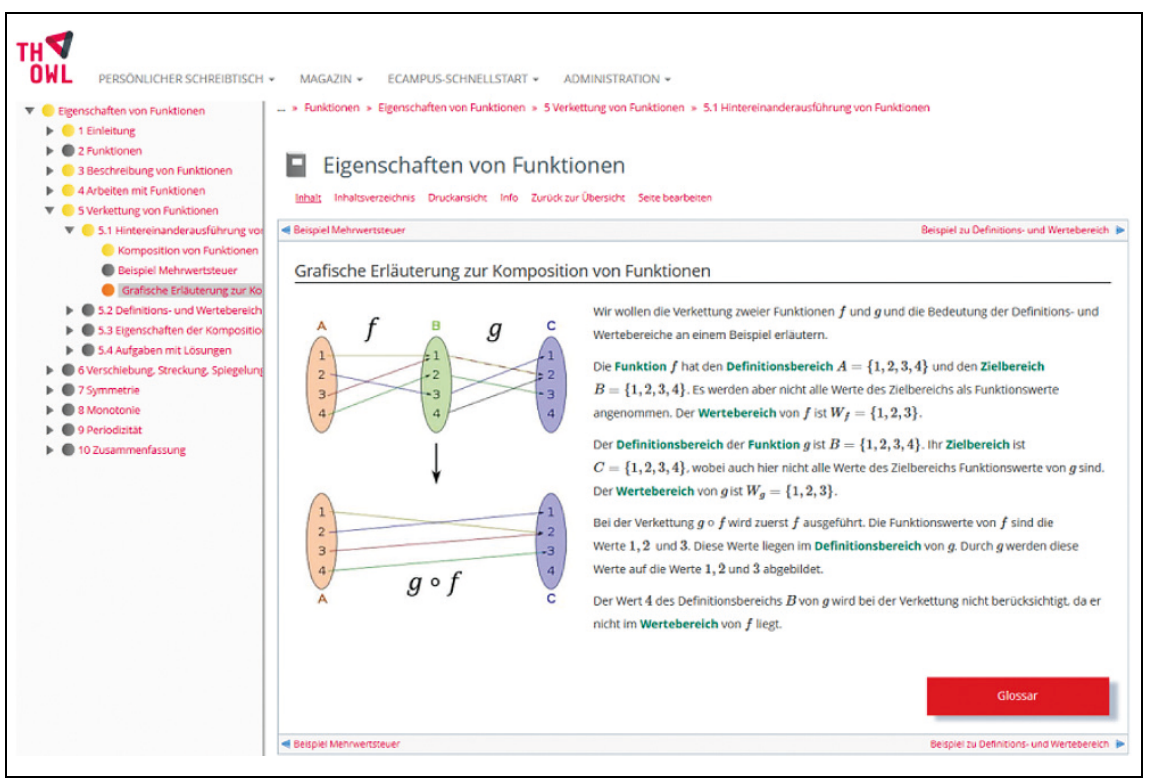

Abbildung 3: Beispieleinsicht des Lernmoduls Funktionen

gebung erhalten die Lernenden durch die oben aufgeführten Tests (vorneweg der Diagnostische Einstiegstest) zunächst eine erste Orientierung über die Lerninhalte, um dem Phänomen „Lost in Hyperspace“ entgegenzuwirken (ebd.). Die Tests sind zudem ein Instrument zur Reflexion und Evaluation des Lernprozesses, wodurch unter anderem die Selbsteinschätzung der Teilnehmenden gefördert werden soll.

Daneben haben Richardson, Abraham und Bond (2012) festgestellt, dass unter anderem ein hohes Selbstwirksamkeitsgefühl sowie die Fähigkeit zum Zeitmanagement ,höhere Zusammenhänge zu akademischem Erfolg aufweisen, als beispielsweise Intelligenz oder sozioökonomischer Status“ (Bellhäuser und Schmitz 2013, S. 345). Daher soll durch das vorgesehene Konzept die Selbstwirksamkeit der Teilnehmenden gestärkt werden. Häufig nehmen Lernende eine passive, konsumierende Haltung ein und realisieren erst zu einem späteren Zeitpunkt, dass sie durch andere Personen demonstrierte Inhalte nicht eigenständig beherrschen. Besonders in der Mathematik ist das eigenständige Anwenden der Inhalte unerlässlich, um die Inhalte zu verstehen und das eigene Kompetenzgefühl zu steigern (Bescherer und Spannagel 2008). Neben den Trainingstests stand den Studierenden 2019 dafür der Freie Trainingsplatz zur Verfügung. Mit Hilfe der knapp 300 Fragen zu den Lernzielorientierten Kursen konnten sie ihr neues Wissen anwenden und festigen. Bei falscher Beantwortung der Fragen erhielten die Teilnehmenden Hinweise, in welchem Lernmodul sie die entsprechenden Lerninhalte finden, um 
die Fragen richtig beantworten zu können. Der Freie Trainingsplatz wird aufgrund der Erweiterung der Lerninhalte ab 2020 durch adaptive Trainings ersetzt.

Wichtig für das selbstregulierte Lernen und das Kompetenzerleben sind des Weiteren Erfolgserlebnisse. Daher finden die Studierenden während der Bearbeitung der Inhalte im Vorkurs Hinweise und Tipps aus überfachlichen Themengebieten, die sie in der Ausgestaltung und im Erfolg ihres Lernprozesses unterstützen sollen. Diese Tipps und Hinweise sind jeweils zu Beginn und/oder zum Abschluss eines Lernmoduls platziert. Zum einen erhalten die Teilnehmenden praktische Hinweise und Reflexionsangebote, die direkt umgesetzt werden können. Dazu zählen zum Beispiel der Tipp, einmal gut durchzulüften, oder die Frage, wie gut die Bearbeitung des letzten mathematischen Lernziels aus eigener Sicht verlaufen ist.

Zum anderen stehen den Studienanfänger*innen Hinweise zu überfachlichen Lernmodulen zur Verfügung (siehe Kapitel 16). Diese behandeln Themen, wie Methoden des Zeit- und Selbstmanagements, Ratschläge zum Aufgabenmanagement und der Gestaltung des eigenen Lernprozesses, Tipps zur Kommunikation mit Mitstudierenden und Dozent*innen, Hinweise zur Selbstmotivation sowie zum Umgang mit Fehlern und Schwierigkeiten.

\subsection{Digitale Unterstützungsangebote}

Parallel zum individuellen Lernprozess entsteht eine Fähigkeitsmatrix, die einen Überblick der Fortschritte abbildet (siehe Kapitel 6). Dadurch ist für die Studienanfänger*innen ersichtlich, welche Lernziele sie bereits erreicht haben und welche Kenntnisse weiterhin ausbaufähig sind. Die Entwicklung der Studierenden wird zusätzlich in einem begleitenden E-Portfolio dokumentiert (siehe Kapitel 5).

Zudem haben die Teilnehmenden die Möglichkeit, online Unterstützung zu Lernstrategien oder zu fachlichen Inhalten in Lerngruppen sowie von E-Mentor*innen zu erhalten. E-Mentor*innen sind Studierende höherer Semester, welche beispielsweise in Sprechstunden (online und vor Ort) bei der Nutzung der Lernplattform helfen und Impulse zur Selbstreflexion liefern (siehe hierzu Kapitel 18). Darüber hinaus werden Online-Tutorien zu den überfachlichen Lernmodulen angeboten. Hier werden Themen aus den überfachlichen Lernmodulen (zum Beispiel zum Zeit- oder Aufgabenmanagement) aufgegriffen und zusammen mit einem oder einer erfahrenden Studierenden vertieft. Weitere Information finden die neuen Studierenden außerdem online im „Studyguide“ sowie in dem Forum und Glossar. Der „Studyguide“ ist eine Orientierungs- und Einstiegshilfe auf der Lernplattform eCampus (siehe Kapitel 18). Das Glossar stellt ein ausführliches Nachschlagewerk dar, welches zahlreiche mathematische Begriffe und Formeln erläutert. 


\subsection{Begleitetes Lernen vor Ort}

Neben dem Selbststudium und den dazugehörigen Unterstützungsangeboten können die Erstsemester an betreuten Übungskursen (sogenannten Tutorien) teilnehmen. Angeboten werden die Tutorien an allen drei Standorten der TH OWL und online auf der Lernplattform eCampus. Die Tutorien behandeln jeweils ein Lernziel und finden wiederholt, zu unterschiedlichen Zeiten statt, sodass sich für alle Studierenden die Chance erhöht, daran teilzunehmen. Für Studierende, welche noch nicht vor Ort oder aus anderen Gründen verhindert sind, werden zusätzlich Online-Tutorien angeboten. Die Tutorien dienen der Anwendung, dem Abgleich und der Überprüfung des Gelernten. Dies geschieht durch Klärung von Fragen, Lösen von (Gruppen-)Aufgaben oder Besprechen von Musterlösungen. Die Ausgestaltung der Vorkurse soll neben selbstständigem Lernen zudem Lerngruppen fördern, um dadurch kollaboratives Lernen insgesamt als eine Selbstverständlichkeit an der Hochschule kennenzulernen.

Begleitet wird das Tutorium durch eine Lehrperson, welche die Rolle einer Lernbegleitung übernimmt. Sie berät bei der Lösungsfindung, beobachtet und moderiert und regt die Studierenden zur Kommunikation, Kooperation und Reflexion an. Die Lehrenden erhalten zuvor in einem Workshop die nötigen Informationen zu der Lernplattform, den mathematischen optes-Materialien und zum didaktischen Konzept. Die Lehrenden, welche die Online-Tutorien betreuen, werden zusätzlich mit der Nutzung der Web-Konferenz-Software Adobe Connect sowie der Grafik-Tablets vertraut gemacht. Diese Tablets stellen eine digitale Alternative zur Tafel oder zum Overhead-Projektor dar. Mit ihnen können die Lehrenden (gemeinsame) Lösungswege visualisieren.

Die Teilnahme an einem Tutorium erfordert zunächst eine Anmeldung, welche im eCampus erfolgt. Dort finden die Studierenden Angaben zu den Inhalten, Terminen und Räumlichkeiten der Tutorien sowie dazu, was sie dafür vorzubereiten bzw. mitzubringen haben. Zur Vorbereitung sollen sie die zwei folgenden Fragen zu ihrem Lernstand beantworten: „Mit welcher Aufgabe hatte ich Probleme?“ und „Ich habe folgende Frage:““. Die Beantwortung der Fragen unterstützt zum einen die vorherige Auseinandersetzung mit dem Lernstoff (siehe Inverted-ClassroomModell). Des Weiteren wird die bzw. der Lehrende im Vorfeld darüber informiert, welche konkreten Fragen bzw. Probleme die Studierenden mitbringen, und kann aufgrund dieser Rückmeldung die Veranstaltung planen. Hier wird das Just-intime-teaching-Lehrkonzept aufgegriffen: Aufbauend auf dem Inverted-Classroom-Modell bearbeiten die Lernenden vor dem Präsenztermin die Lerninhalte. Zusätzlich melden die Studierenden der Lehrperson, welche Schwierigkeiten sie im Selbststudium mit dem Lernstoff hatten. Die Lehrperson kann mit diesen Informationen in der Präsenzveranstaltung vorbereitet auf die Anliegen der Studierenden eingehen. Durch diese Vorarbeit werden Zeitressourcen gewonnen, welche für vertieftes Lernen genutzt werden können. Außerdem erfordert die Aufgabe eine 

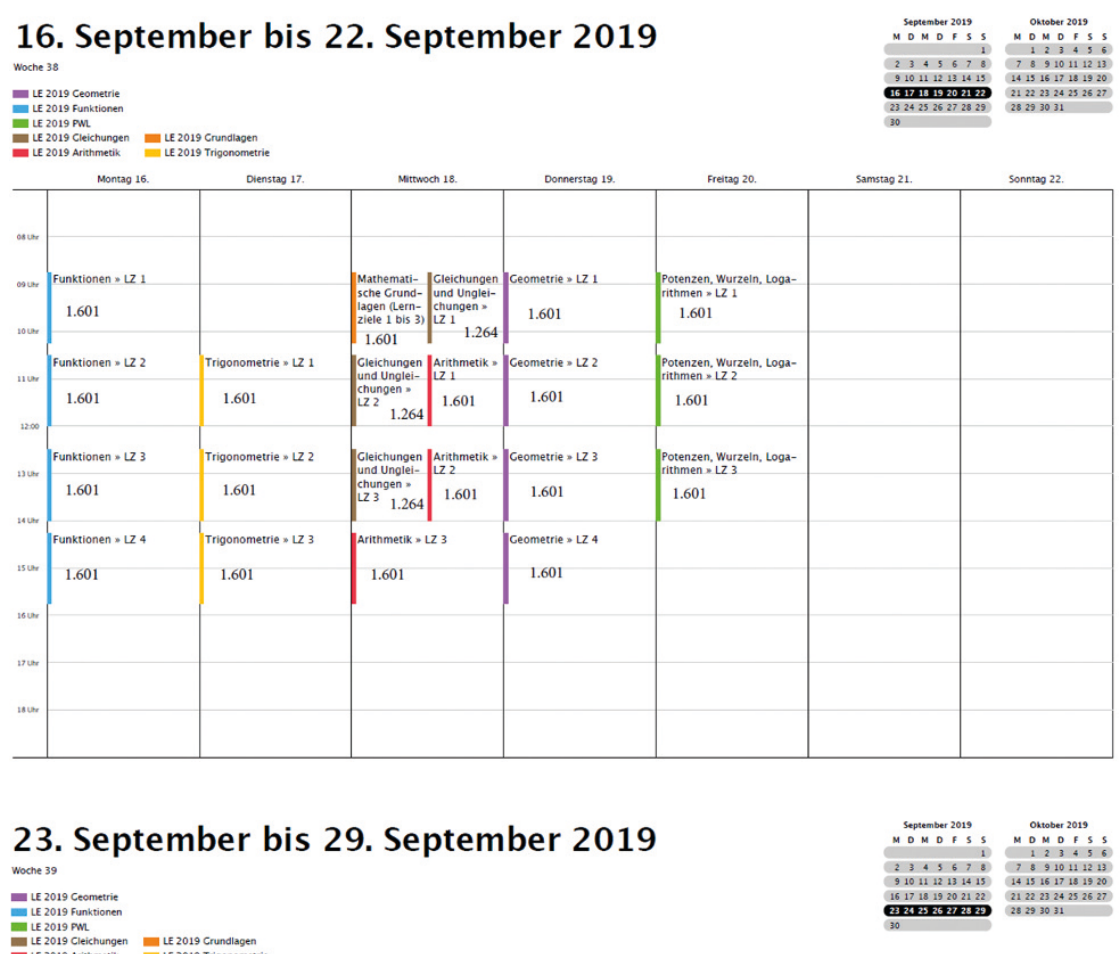

드 2019 PML
LE 2019 Cleichungen
LE 2019 Arithmetil 2019 Crundlagen
LE 2019 Trigonometrie

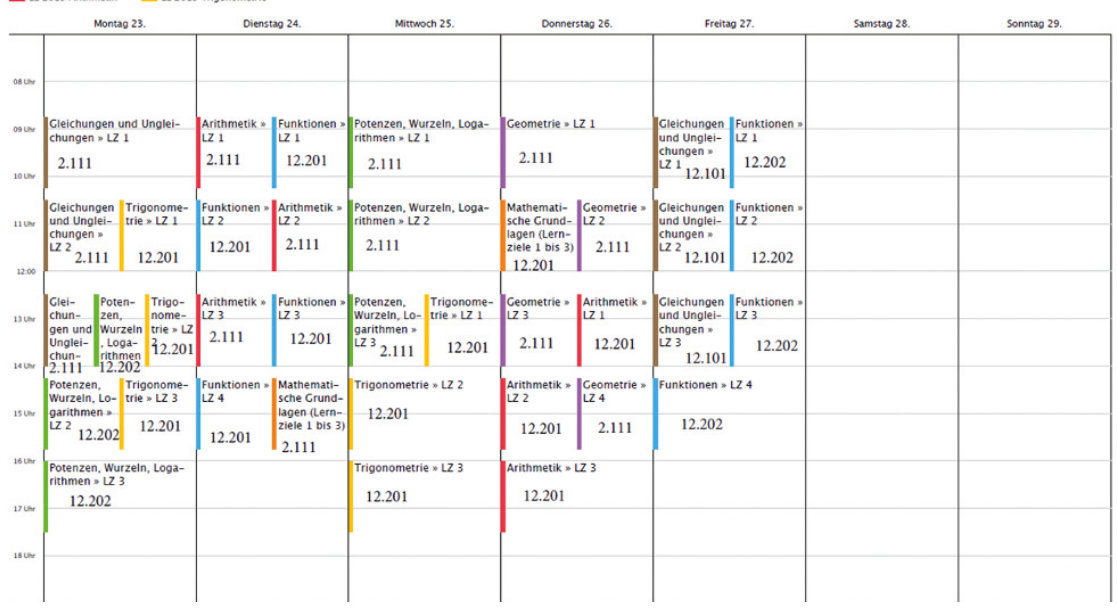

Abbildung 4: Beispielhafte Wochenpläne zu den Tutorien 
aktive Auseinandersetzung der Studienanfänger*innen mit den Lerninhalten, wodurch sie Aspekte ihrer Selbstlernkompetenz trainieren.

Dafür wird, neben den erhöhten Selbststudiumsanteilen, in den Tutorien möglichst von der reinen Präsentation der Inhalte abgesehen, um der passiven, konsumierenden Haltung entgegenzuwirken. Zum Einbeziehen und Aktivieren der Teilnehmenden wird ein Wechsel von Einzel- und Gruppenarbeit sowie Plenum empfohlen. Darüber hinaus können weitere Methoden eingesetzt werden, wie etwa die Think-Pair-Share-Methode, welche die drei oben aufgeführten Methoden kombiniert, oder das Einbinden von Voting-Tools, wie zum Beispiel PINGO oder Mentimeter.

Zum Abschluss des Tutoriums werden die Studierenden gebeten, an dem Expressfeedback, einer kurzen Umfrage zur Zufriedenheit mit dem Lehrangebot, teilzunehmen. Bedauerlicherweise haben sich daran kaum Teilnehmende beteiligt.

Neben den Präsenzveranstaltungen finden zusätzlich regelmäßige OnlineTutorien in einem virtuellen Klassenraum via Adobe Connect statt. Hiermit soll gewährleistet werden, dass Studienanfänger*innen, welche noch nicht vor Ort wohnen oder aus anderen Gründen nicht an den Präsenzterminen teilnehmen können, ebenfalls tutorielle Unterstützung erhalten. Das Online-Tutorium setzt sich wie das Pendant in Präsenz, aus Übungsaufgaben und dem Beantworten von Fragen zusammen. Allerdings sind Online-Tutorien zwar inhaltlich einem Leistungsorientierten Kurs zugehörig, jedoch nicht nach Lernzielen aufgetrennt.

\subsection{Marketing und Motivation der Studierenden}

Aufgrund der Tatsache, dass der Vorkurs an der Technischen Hochschule OWL nicht verpflichtend ist, wird der Kurs über verschiedene Medien beworben. Zunächst erhalten die Studierenden mit ihren Immatrikulationsunterlagen einen Flyer mit den ersten Informationen zum Vorkurs. Weiterführende Informationen sind auf der Vorkurs-Website zu finden. Daneben gibt es verschiedene ausführliche Dokumente, beispielsweise eine FAQ-Liste und eine Anleitung zum Anmeldeprozess. Diese wird für internationale Studierende ebenfalls in englischer Sprache angeboten. Neben den Lesezeichen-Flyern, welche verschiedene Tipps zum Studienstart enthalten, werden die Studierenden über die Social-Media-Kanäle der Hochschule (Facebook und Instagram) auf die Angebote aufmerksam gemacht. Dieses Jahr sollen zudem kurze Videos die Studienanfänger*innen über die Vorkurse informieren. Neben den digitalen Angeboten können sie sich zudem an den Service-Points in der Bibliothek oder telefonisch beraten lassen. Neben Veranstaltungshinweise erhalten die Studierenden verschiedene Informationen per E-Mail. Unter anderem werden sie hierüber für ihre fleißige Teilnahme gelobt oder freundlich auf ihre ausbaufähige Teilnahme hingewiesen. Hierzu wird beispielsweise geprüft, wer noch nicht an dem Einstiegs- oder Abschlusstest teilgenommen oder lediglich ein bis zwei Lernzielorientierte Kurse begonnen hat. 
Zur weiteren Motivation wurden sogenannte Badges verliehen. Badges sind digitale Zertifikate oder Abzeichen, welche den Studierenden eine kurze und prägnante Rückmeldung zu ihren Lernerfolgen geben. Angelehnt an den GamificationAnsatz enthalten sie neben einem ansprechenden Bild detaillierte Informationen über die erbrachte Leistung.

Da für das Selbstwirksamkeitsgefühl ebenso die soziale Eingebundenheit von großer Bedeutung ist (Bescherer und Spannagel 2008) und um eventuelle Einstiegshürden abzubauen, wurde 2019 ein lockerer Beratungsnachmittag mit gemeinsamen Grillen, angeboten. Hier wurden die Teilnehmenden über die Vorkurse und die vielfältigen Unterstützungsangebote zum Studienstart informiert und konnten in lockerer Atmosphäre und durch geführte Methoden ihre Kommiliton*innen sowie die Mitarbeitenden des Vorkurs-Teams kennenlernen. Ein weiterer Mehrwert stellte die Gelegenheit zum Netzwerken dar, welche genutzt wurde, um Mitbewohner*innen, Mitfahrgelegenheiten und vor allem Lerngruppen zu finden. Durch das Kennenlernen, die nützlichen Informationen und die schöne Atmosphäre beim Grillen sollten die Studierenden für die Vorkurse sowie für ihren Start an der TH OWL motiviert werden. 2020 sollen zusätzlich gemeinsame Einstiegsveranstaltungen stattfinden, in denen zu Beginn einer Woche der Vorkurs vorgestellt wird. Dies beinhaltet den organisatorischen Ablauf des Kurses, die optimale Verwendung des Materials, erste Tipps zum selbstständigen Lernen sowie Hinweise zu den Unterstützungsangeboten und der Bildung von Lerngruppen.

\subsection{Evaluation}

Für die Evaluation der Vorkurse 2019 wurden zum einen die Daten zur Nutzung der Lernplattform eCampus genutzt. Des Weiteren wurden die Teilnehmenden mittels eines Fragebogens (ebenfalls auf der Lernplattform) zum Vorkurs befragt.

Von den mehr als 1500 Neuzugängen im Jahr 2019 haben sich knapp die Hälfte zu den Vorkursen angemeldet. Die Hälfte der zum Abschluss des Kurses befragten Studierenden haben den Mathematik-Vorkurs ein- bis drei Mal pro Woche genutzt. Knapp 33\% nutzen ihn häufiger als drei Mal pro Woche (TN 80). Knapp 35\% stimmten eher der Aussage zu, dass sie mit den angebotenen Lerninhalten die angegebenen Lernziele erreichen konnten (20\% stimme voll und ganz zu; 23\% stimme teilweise zu; TN 79). Außerdem ergab die Befragung, dass die meisten Studierenden durch den Vorkurs dazu angeregt wurden, sich intensiver mit den mathematischen Inhalten ihres Studiums auseinanderzusetzen (23\% stimme voll und ganz zu, 38\% stimme eher zu; TN 74). Zudem bestätigten die Befragten die Wirksamkeit der Reflexions- und Kontrollinstrumente für das Selbststudium, indem die meisten angaben, dass sie während des Vorkurses jederzeit ihren Kenntnisstand selbstständig überprüfen konnten (27\% stimme voll und ganz zu, $40 \%$ stimme eher zu; TN 74). 


\section{Literatur}

Bellhäuser, H. \& Schmitz, B. (2013). Förderung selbstregulierten Lernens für Studierende in mathematischen Vorkursen - ein web-basiertes Training. In I. Bausch, R. Biehler, P. D. R. Bruder, P. R. Fischer, R. K. Hochmuth, W. Koepf, S. Schreiber \& T. Wassong (Hrsg.), Mathematische Vor- und Brückenkurse: Konzepte, Probleme und Perspektiven. Konzepte und Studien zur Hochschuldidaktik und Lehrerbildung Mathematik (Bd. 2, S. 327-342). Wiesbaden: Springer Fachmedien.

Bescherer, C. \& Spannagel, C. (2008). Aktivierendes Mathematik-Lernen zum Studienbeginn. In E. Vásárhelyi (Hrsg.), Beiträge zum Mathematikunterricht 2008 (S. 329-332). Münster: WTM.

Richardson, M., Abraham, C. \& Bond, R. (2012). Psychological correlates of university students' academic performance: A systematic review and meta-analysis. Psychological bullet, 138(2), 353-387.

Tolks, D., Schäger, C., Raupach, T., Kruse, L., Sarikas, A., Gerhardt-Szép, S., Kllauer, G., Lemos, M., Fischer, M., Eichner, B., Sostmann, K. \& Hege, I. (2016). Eine Einführung in die Inverted/Flipped-Classroom-Methode in der Aus- und Weiterbildung in der Medizin und den Gesundheitsberufen. GMS Journal for Medical Education, Vol. 33(3). Düsseldorf.

Open Access Dieses Kapitel wird unter der Creative Commons Namensnennung 4.0 International Lizenz (http://creativecommons.org/licenses/by/4.0/deed. de) veröffentlicht, welche die Nutzung, Vervielfältigung, Bearbeitung, Verbreitung und Wiedergabe in jeglichem Medium und Format erlaubt, sofern Sie den/ die ursprünglichen Autor(en) und die Quelle ordnungsgemäß nennen, einen Link zur Creative Commons Lizenz beifügen und angeben, ob Änderungen vorgenommen wurden.

Die in diesem Kapitel enthaltenen Bilder und sonstiges Drittmaterial unterliegen ebenfalls der genannten Creative Commons Lizenz, sofern sich aus der Abbildungslegende nichts anderes ergibt. Sofern das betreffende Material nicht unter der genannten Creative Commons Lizenz steht und die betreffende Handlung nicht nach gesetzlichen Vorschriften erlaubt ist, ist für die oben aufgeführten Weiterverwendungen des Materials die Einwilligung des jeweiligen Rechteinhabers einzuholen.

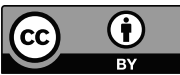

